\title{
Multicenter Prospective Trial of Stent Placement in Patients with Symptomatic High-Grade Intracranial Stenosis
}

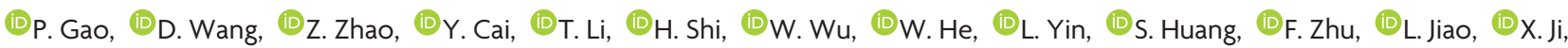 \\ (1) A.I. Qureshi, and (1)F. Ling
}

\begin{abstract}
BACKGROUND AND PURPOSE: On the basis of the high 1-month stroke and/or death (14.7\%) rates associated with stent placement in the Stenting versus Aggressive Medical Management for Preventing Recurrent Stroke in Intracranial Stenosis trial, modifications in patient selection and procedural aspects for intracranial stent placement have been recommended. We performed a multicenter prospective single-arm trial to determine whether such modifications would result in lower rates of periprocedural stroke and/or death.
\end{abstract}

MATERIALS AND METHODS: The study enrolled patients with recent transient ischemic attack or ischemic stroke (excluding perforator ischemic events) related to high-grade (70\%-99\% in severity) stenosis of a major intracranial artery. Patients were treated by using angioplasty and self-expanding stents 3 weeks after the index ischemic event at 1 of the 10 high-volume centers in China. An independent neurologist ascertained the occurrence of any stroke and/or death within 1 month after the procedure.

RESULTS: A total of 100 consecutive patients were recruited. The target lesions were located in the middle cerebral artery (M1) $(n=38$, $38 \%)$, intracranial internal carotid artery $(n=17,17 \%)$, intradural vertebral artery $(n=18,18 \%)$, and basilar artery $(n=27,27 \%)$. The technical success rate of stent deployment with residual stenosis of $<50 \%$ was $100 \%$. The overall 1 -month stroke and/or death rate was $2 \%(95 \%$ confidence interval, $0.2 \%-7.0 \%$ ). Two ischemic strokes occurred in the pontine region (perforator distribution) in patients following angioplasty and stent placement for basilar artery stenosis.

CONCLUSIONS: The results of this prospective multicenter study demonstrated that modifications in patient selection and procedural aspects can substantially reduce the 1-month stroke and/or death rate following intracranial stent placement.

ABBREVIATION: SAMMPRIS = Stenting versus Aggressive Medical Management for Preventing Recurrent Stroke in Intracranial Stenosis

ntracranial atherosclerosis is an important cause of cerebral ischemia with a relatively high prevalence in Chinese patients. ${ }^{1}$ The Chinese Intracranial Atherosclerosis study reported a prevalence of intracranial stenosis of $46.6 \%$ among 2864 consecutive

Received August 18, 2015; accepted after revision December 16

From the Department of Neurosurgery (P.G., F.Z., L.J., X.J., F.L.), Xuanwu Hospital, Capital Medical University, Beijing, China; Department of Neurosurgery (D.W.), Beijing Hospital of the Ministry of Health, Beijing, China; Department of Neurosurgery (Z.Z.), Tangdu Hospital, the Fourth Military Medical University, Xi'an, Shaanxi Province, China; Department of Neurology (Y.C.), the 306 Hospital of the People's Liberation Army, Beijing, China; Department of Intervention (T.L.), Henan Provincial People's Hospital, Zhengzhou, Henan Province, China; Department of Neurosurgery (H.S.), the 1st Affiliated Hospital of Harbin Medical University, Harbin, Heilongjiang Province, China; Department of Neurology (W.W.), Qilu Hospital of Shandong University, Ji'nan, Shandong Province, China; Department of Neurosurgery (W.H.), the 2nd Affiliated Hospital of Guangzhou Medical College, Guangzhou, Guangdong Province, China; Department of Neurosurgery (L.Y.), Tianjin Huanhu Hospital, Tianjin, China; Department of Neurosurgery (S.H.), Guangdong Province Hospital of Traditional Chinese Medicine, Guangzhou, Guangdong Province, China; and Zeenat Qureshi Stroke Institute (A.I.Q.), St. Cloud, Minnesota.

This study was supported by National Health and Family Planning Commission of the People's Republic of China (2011BAI08B04) and the National Natural Science Foundation of China (81200910). This trial been registered and released in ClinicalTrials.gov with the ID NCT01763320.
Chinese patients with cerebral ischemia. ${ }^{2}$ Patients with ischemic symptoms related to high-grade intracranial stenosis (70\%-99\%) have an almost $20 \%$ risk of recurrent stroke within 1 year despite antithrombotic treatment. ${ }^{3}$ Therefore, intracranial angioplasty and stent placement have been recommended to reduce the rate of recurrent ischemic events. ${ }^{4-7}$ However, the Stenting versus Aggressive Medical Management for Preventing Recurrent Stroke in Intracranial Stenosis (SAMMPRIS) trial $^{8}$ was prematurely terminated due to excessively high 1-month stroke and/or death rates in patients randomized to intracranial stent placement. At the time of the Data Safety Monitoring review, $14.7 \%$ of patients treated with angioplasty combined with stent placement experienced a stroke or died within 1 month after enrollment compared with $5.8 \%$ of patients treated with medical therapy alone, a highly

Please address correspondence to Liqun Jiao, MD, PhD, Department of Neurosurgery, Xuanwu Hospital, Capital Medical University, 45 Changchun St, Beijing 100053, China; e-mail: liqunjiao@sina.cn

- Indicates open access to non-subscribers at www.ajnr.org

三 Indicates article with supplemental on-line tables.

http://dx.doi.org/10.3174/ajnr.A4698 
1) Eligible patients between 30 and 80 years of age; intracranial arterial stenosis will not be considered if related to the following: nonatherosclerotic factors: arterial dissection, Moyamoya disease; vasculitic disease; herpes zoster, varicella zoster, or other viral vasculopathies; neurosyphilis; any other intracranial infection; any intracranial stenosis associated with CSF pleocytosis; radiation-induced vasculopathy; fibromuscular dysplasia; sickle cell disease; neurofibromatosis; benign angiopathy of the central nervous system; postpartum angiopathy; suspected vasospastic process; and suspected recanalized embolus

2) Symptomatic intracranial stenosis: presenting with TIA or stroke within the past 12 months attributed to $70 \%-99 \%$ stenosis of a major intracranial artery (internal carotid artery, MCA [M1], vertebral artery, or basilar artery)

3) Degree of stenosis: $70 \%-99 \%$ severity confirmed by catheter angiography for enrollment in the trial

4) Remote infarctions on MRI were acceptable, which could be accounted for by the occlusion of the terminal cortical branches or hemodynamic compromise (perforator strokes excluded); perforator strokes due to perforator occlusion are defined as basal ganglia or brain stem/thalamus infarction related with middle cerebral artery or basilar artery stenosis

5) Expected ability to deliver the stent to the lesion

6) All patients should be treated beyond a duration of 3 weeks from the latest ischemic symptom onset

7) No recent infarctions identified on MRI (indicated as high signals on DWI series) at enrollment

8) No massive cerebral infarction (more than one-half of the MCA territory), intracranial hemorrhage, epidural or subdural hemorrhage, and intracranial brain tumor on CT or MRI

9) $\mathrm{mRS}$ score of $\leq 2$

10) Target vessel reference diameter must be measured at $2.00-4.50 \mathrm{~mm}$; target area of stenosis is $\leq 14 \mathrm{~mm}$ in length

11) No childbearing potential or has a test negative for pregnancy within the week prior to study procedure; female patients had normal menses in the past 18 months

12) Patient is willing and able to return for all follow-up visits required by the protocol

13) Patients understand the purpose and requirements of the study and have signed an informed consent form

significant difference. ${ }^{1,9}$ The 1-month stroke and/or death rate was much higher than the $6.6 \%, 4.5 \%$, and $6.5 \%$ rates in the prospective Stenting of Symptomatic Atherosclerotic Lesions in the Vertebral or Intracranial Arteries (SSYLVIA) study, ${ }^{10}$ Wingspan study, ${ }^{11}$ and Apollo Stent for Symptomatic Atherosclerotic Intracranial Stenosis (ASSIST) study, ${ }^{12}$ respectively. Possible reasons for the disproportionately high rates of 1-month stroke and/or death included a very short time interval between the index ischemic event and the procedure, lack of stratification by ischemic event type, and less rigorous operator-experience requirements. ${ }^{13}$

The Food and Drug Administration in March 2012 announced that the Wingspan stent system (Stryker Neurovascular, Kalamazoo, Michigan) continues to remain an option for patients with recurrent stroke despite medical management who have not had any new stroke symptoms within 7 days before treatment with the Wingspan. The decision was based on review of the SAMMPRIS trial and the clinical study data supporting humanitarian device exemption approval data, supplemented by the opinions of an advisory panel of experts. The manufacturer, Stryker Neurovascular, was also required to enhance its physician training program for the Wingspan stent. Another expert panel concluded that the SAMMPRIS trial data support modification but not discontinuation of the use of intracranial angioplasty and/or stent placement for intracranial stenosis. ${ }^{13}$ The panel further recommended proceeding with another clinical trial with appropriate modifications in design based on lessons learned from the SAMMPRIS trial to avoid unnecessary elimination of a potentially beneficial treatment in appropriately selected patients.

On the basis of the above-mentioned considerations, a multicenter prospective single-arm trial with independent outcome ascertainment was undertaken to determine whether such modifications will result in lower rates of periprocedural 1-month stroke and/or death in patients treated with intracranial stent placement.

\section{MATERIALS AND METHODS Patient and Site Selection}

The study was an investigator-initiated, government-funded, prospective, multicenter registration trial that was conducted at 10 clinical sites in China. Patients who had experienced a recent TIA or nondisabling ischemic stroke (modified Rankin Scale score, $\leq 2)$ caused by high-grade stenosis (70\%-99\% in severity) of a major intracranial artery (middle cerebral artery [M1], intracranial internal carotid artery, intradural vertebral artery, and basilar artery) were eligible. Conventional angiography was used to quantitate the severity of stenosis by using the Warfarin-Aspirin Symptomatic Intracranial Disease Study criterion. ${ }^{3}$ Patients who had ischemic symptoms within the most recent 3 weeks were excluded. Patients with perforator strokes only were not considered candidates for stent placement. Here, perforator strokes due to perforator occlusion are defined as basal ganglia or brain stem/thalamus infarction related to middle cerebral artery or basilar artery stenosis. The inclusion and exclusion criteria for the trial are provided in Table 1 and On-line Table 1. This study is registered in the ClinicalTrials.gov with ID NCT01763320 (China Angioplasty and Stenting for Symptomatic Intracranial Severe Stenosis).

The 10 participating sites were selected on the basis of the volume of procedures performed. At each site, the annual volume of intracranial angioplasty and stent placement procedures performed was $>30$ procedures for the past 3 years. At each site, the study team consisted of a neurologist, a neurosurgeon, a neuroradiologist, and a research coordinator. The study protocol was reviewed and approved by a central Data Safety Monitoring Board and subsequently by the local institutional review board. Each patient signed a written informed consent before the procedure. Relevant data were recorded on a standard case reporting form.

\section{Treatment Protocol}

The patients were placed on aspirin, $100 \mathrm{mg}$ daily, and clopidogrel, $75 \mathrm{mg}$ daily, for 3-5 consecutive days before the proce- 
dure. The procedure was performed with the patient under general anesthesia in all except 1 case. The case was typically performed via a transfemorally placed 6F-long sheath or guiding catheter. The intracranial stenotic lesion was traversed by using a standard 0.014-inch microcatheter-microguidewire system by using high-magnification fluoroscopic road-mapping techniques. The microcatheter was exchanged over a 300-cm-long 0.014-inch microguidewire for a Gateway angioplasty balloon (Stryker Neurovascular). After angioplasty, the Gateway angioplasty balloon catheter was exchanged over the existing 0.014 -inch microguidewire for a self-expanding nitinol Wingspan stent delivery system. In general, the Wingspan stent diameter was $0.5-1.0 \mathrm{~mm}$ greater than the target artery and was deployed to extend at least $3 \mathrm{~mm}$ on either side of the lesion. ${ }^{11}$ The Wingspan was deployed across the lesion by using the standard technique of outer containing catheter withdrawal. If the residual stenosis after Wingspan stent deployment was $>50 \%$ in severity, the study protocol allowed postdilation with a new angioplasty balloon catheter. Technical success was determined by successful placement of the stent across the lesion and residual stenosis of $<50 \%$ on postprocedural angiography. Throughout the procedure, intravenous heparin boluses were given to maintain the activated clotting time between 250 and 300 seconds.

The protocol required frequent measurements of blood pressure during the procedure and at least 1 measurement every half an hour during the next 24 hours while the patient was monitored in an intensive care unit. The systolic blood pressure was maintained between 100 and $120 \mathrm{~mm} \mathrm{Hg}$ for 24 hours after the procedure. The patient was continued on aspirin, $100 \mathrm{mg}$ daily, and clopidogrel, $75 \mathrm{mg}$ daily, for the next 90 days and subsequently on aspirin alone. Concurrent risk-factor modification was undertaken, consisting of normalizing low-density lipoprotein (statins, target low-density lipoprotein of $<2.58 \mathrm{mmol} / \mathrm{L}$ [100 mg/dL]), hypertension (systolic pressure of $<140 \mathrm{~mm} \mathrm{Hg}$ and diastolic pressure of $<90 \mathrm{~mm} \mathrm{Hg}$ ), and glycemic status (in patients with diabetes, the hemoglobin A1c level was checked with a target level of $<6.5 \%$ ), and lifestyle modification. ${ }^{14}$

\section{End Point Definition and Ascertainment}

Primary end points included any stroke and/or death within 1 month. A stroke was defined as a sudden-onset neurologic deficit that persisted for at least 24 hours and could be ischemic or hemorrhagic in nature. Ischemic stroke was further defined as a new focal neurologic deficit that was not associated with an intracranial hemorrhage on brain CT or MR imaging. Hemorrhagic stroke was defined as parenchymal, subarachnoid, or intraventricular hemorrhage detected by CT or MR imaging that resulted in a stroke (as defined above) or seizure. The hemorrhage was classified as asymptomatic if symptoms or signs were temporary (lasted $<24$ hours) without any seizures. ${ }^{15}$ Asymptomatic strokes were considered adverse events but were not included as primary end points.

At each site, the site-designated neurologist who was not part of the treating team ascertained the clinical outcomes within the 1-month follow-up. The neurologist along with the study coordinator performed each follow-up visit and collected the data regarding study end points.
Table 2: Demographic and clinical characteristics of the patients treated in the single-arm trial

\begin{tabular}{lc}
\hline \multicolumn{2}{c}{ Baseline Demographics } \\
\hline Age (median) (IQR) & $56.0(49-63)$ \\
Men (No.) (\%) & $73(73 \%)$ \\
Risk factors & \\
$\quad$ Hypertension (No.) (\%) & $61(61 \%)$ \\
Hyperlipidemia (No.) (\%) & $15(16 \%)$ \\
$\quad$ Diabetes mellitus (No.) (\%) & $25(28 \%)$ \\
$\quad$ History of cigarette smoking (No.) (\%) & $44(44 \%)$ \\
History of alcoholism (No.) (\%) & $28(28 \%)$ \\
Ischemic stroke as qualifying event (No.) (\%) & $50(50 \%)$ \\
NIHSS sores at admission (median) (IQR) & $0(0-1)$ \\
mRS scores at admission (median) (IQR) & $0(0-1)$ \\
Barthel Index at admission (median) (IQR) & $100(100-100)$ \\
Angiographic characteristics & \\
Preprocedure: average degree of stenosis (\%) & $82.7 \pm 8.9$ \\
Location & \\
Basilar artery & $27(27 \%)$ \\
Intracranial internal carotid artery & $17(17 \%)$ \\
$\quad$ Middle cerebral artery & $38(38 \%)$ \\
Intradural vertebral artery & $18(18 \%)$ \\
Postprocedure: average stenosis (\%) & $13.5 \pm 10.2$ \\
\hline Note:-IQR indicates interquartile range.
\end{tabular}

Note:-IQR indicates interquartile range.

\section{Statistical Analysis}

The statistical methods used were predominantly descriptive. Continuous data were presented as means (with SDs), and categoric data were presented as percentages. For selected percentages, $95 \%$ confidence intervals were calculated by using the binomial (Clopper-Pearson) "exact" method. ${ }^{16}$

\section{RESULTS}

From July 2013 to March 2014, 10 participating sites evaluated 235 consecutive patients with symptomatic high-grade intracranial stenosis or occlusion (70\%-100\% in severity by angiography). Among them, 135 patients were finally excluded from the study because of the following aspects: 1) Patients did not meet the inclusion criteria; 2) refused to accept endovascular stent placement; and 3) had chronic occlusion of target major intracranial artery. A total of 100 intracranial lesions were treated in 100 enrolled patients (median age, 56 years; $73 \%$ were men) (Table 2 ). All procedures were a combination of angioplasty followed by stent placement performed with the patient under general anesthesia (except 1 case) via the femoral approach. None of the patients required additional postdilation or $>1$ stent placement. Angioplasty and stent placement were performed in the following locations: 27 (27\%) in the basilar artery, 17 (17\%) in the intracranial internal carotid artery, 38 (38\%) in the middle cerebral artery, and $18(18 \%)$ in the intradural vertebral artery. The technical success rate was $100 \%$. The mean severity of preprocedural stenosis was $82.7 \% \pm 8.9 \%$ and postprocedure stenosis was $13.5 \% \pm$ $10.2 \%$ (Table 2).

The overall 1-month stroke and/or death rate was $2 \%(95 \%$ confidence interval, $0.2 \%-7.0 \%$ ). Ischemic stroke occurred in the distribution of the perforating arteries (pontine) in 2 patients (On-line Table 2), both of whom had undergone the procedure for high-grade basilar artery stenosis. Both of them had midpontine infarctions on the postprocedure MR imaging. One patient developed hemiparesis and ataxia within 24 hours after the procedure. The other patient developed hemiplegia and facial paral- 
ysis (central) 72 hours after the procedure. The patients were treated with anticoagulation and intravenous hypervolemic hemodilution. Both patients had good recovery (mRS of 0 and 1 at 30-day follow-up, respectively). None of the remaining 98 patients had any new neurologic deficits or died within the 30-day visit. No hemorrhagic stroke, myocardial infarction, or severe adverse events were observed in this study.

\section{DISCUSSION}

We observed a high technical success rate and low rate of 1-month stroke and/or death in patients with high-grade intracranial stenosis treated with intracranial stent placement within this prospective multicenter study. The study was designed after the completion of the SAMMPRIS trial and incorporated modifications in protocol from observations derived from trial results and subsequent expert recommendations. ${ }^{8}$ Several factors may have contributed to the more favorable short-term results observed within the current study. Restriction of patient recruitment to high-volume centers and modifications in patient selection were probably important factors.

In the SAMMPRIS trial, 220 procedures were performed in 50 sites in the United States during 29 months with an average of $<2$ procedures at each site per year. ${ }^{8}$ Such recruitment patterns suggest that either familiarity with the protocol or even operator experience differed from that in our study, which treated 30 patients per year at each site on an average. To participate as an interventionalist in the SAMMPRIS trial, the operator was required to demonstrate previous experience with 20 intracranial angioplasty/stent procedures, of which at least 3 procedures were performed with the Wingspan or Neuroform stent system (Stryker Neurovascular). ${ }^{8}$ In the current study, an annual volume of $>30$ intracranial stent procedures sustained during the past 3 years was required. Our results are comparable with the recent data (1month stroke and/or death rate of $4.4 \%-6.2 \%$ ) derived from some high-volume centers ( $>100$ cases per year). ${ }^{17-19}$ A retrospective analysis of 96 patients treated with intracranial angioplasty and stent placement at 3 university-affiliated institutions in the United States reported that the overall 1-month stroke and/or death rate was $7.2 \%$ in the 69 SAMMPRIS-eligible group and $7.4 \%$ in the 27 SAMMPRIS-ineligible group. ${ }^{20}$ The 30 -day stroke and/or death rate was $3.3 \%$ and $10.2 \%$ in the SAMMPRIS-eligible, angioplasty-treated subgroup and the stent-treated subgroup, respectively.

Patient selection, particularly exclusion of patients with recent ischemic events and those with perforating artery ischemic stroke (in specific contrast to SAMMPRIS) may have contributed to the favorable short-term results in our trial. Our trial recruited patients who had experienced an index ischemic event at least 3 weeks before recruitment, which is longer than the recommended 7-day interval (range, 7-19 days) in the SAMMPRIS trial. The longer time interval may have allowed plaque stabilization and spontaneous lysis of overlying thrombus and probably also reduced the risk of hemorrhagic transformation for patients with recent ischemic stroke ( $<3$ weeks). ${ }^{17,21-23}$ An analysis of the National Institutes of Health Multicenter Wingspan Intracranial Stent Registry Study found that stent placement performed within 10 days of a qualifying ischemic event was associated with a higher rate of 30-day stroke and/or death compared with procedures performed after 10 days ( $8 \%$ versus $17 \%, P=.06) .{ }^{21,24}$ In the SAMMPRIS trial, the rates of ischemic stroke, symptomatic hemorrhagic stroke, or any death within 1 month were $15.7 \%$ and $13.8 \%$ in the patients enrolled within 7 days or after 7 days of their qualifying event, respectively. ${ }^{15}$ Exclusion of patients with recent ischemic stroke may also exclude those with the highest risk of an ischemic event recurrence; therefore, the benefit of stent placement in the reduction of stroke recurrence may also be diminished.

We included patients with distal hypoperfusion and/or cortical involvement. The exclusion of patients with perforating artery ischemic stroke may have reduced the occurrence of this type of stroke postprocedurally. ${ }^{25}$ However, in a post hoc analysis of the SAMMPRIS trial, the rate of postprocedural ischemic events was not higher among those recruited due to perforating artery ischemic stroke $(0 \%)$ compared with those with TIAs $(8.9 \%)$ or nonperforating artery ischemic strokes $(14.3 \%) .{ }^{15}$ Certain procedure-related factors such as clopidogrel load $(\approx 10 \%)$ and poststent angioplasty $(\approx 10 \%)$ performed in the SAMMPRIS trial were avoided in the current study and may have made some contribution to the differences in adverse event rates.

Two additional aspects that could improve the results of the intracranial angioplasty and stent placement are improvement in device design and point-of-care testing for assessing the magnitude of platelet inhibition with antiplatelet medication. Although the self-expandable Wingspan stent with the over-the-wire technique was widely adapted because of the relative ease of delivery over balloon-expandable stents with the rapid-exchange technique; however, the effectiveness of the self-expanding stent in restoring lumen diameter and preventing restenosis has been questioned. Although the radial force exerted by the Wingspan is superior to that of other self-expandable stents such as the Enterprise (Codman \& Shurtleff, Raynham, Massachusetts) and Neuroform stents, it is not comparable with that of balloon-expanding stents. A new generation of balloon-expanding stents with a rapid-exchange platform may result in superior technical results. We did not perform point-of-care testing to guide antiplatelet treatment in our cohort of patients. Point-of-care testing was introduced because considerable differences can be observed within individuals in regard to platelet inhibition with the same doses of aspirin and clopidogrel. ${ }^{26}$ Such assessment may allow the use of higher doses of clopidogrel and intravenous glycoprotein IIb/IIIa inhibitors among those with inadequate response (resistance) to standard doses of antiplatelet medication.

The low rate of adverse events following intracranial angioplasty and stent placement in our trial raises the question of the superiority of such a procedure over intense medical treatment for high-grade symptomatic intracranial stenosis. Intensive medical therapy in the SAMMPRIS trial consisting of aspirin, 325 $\mathrm{mg}$ /day, for entire follow-up; clopidogrel, $75 \mathrm{mg}$ per day for 90 days after enrollment; and aggressive risk-factor management (targeting blood pressure $<130 / 80 \mathrm{~mm} \mathrm{Hg}$ and low-density lipoprotein concentration of $<70 \mathrm{mg} / \mathrm{dL}$ ) had reduced the 30 -day stroke and/or death rate to $5.8 \%$, which was substantially lower than the estimated rate of $10.7 \%$ based on historical controls. ${ }^{8}$ 
Chaudhry et $\mathrm{al}^{27}$ reported that $\mathrm{a} \leq 3.8 \% 1$-month rate of stroke and/or death was required to achieve a $35 \%$ relative risk reduction of the primary end point (composite of 1-month stroke and/or death and ipsilateral stroke beyond 1 month) among the intracranial stent-treated group compared with the medically treated group at 1-year follow-up, as specified by the superiority threshold within the SAMMPRIS hypothesis.

One of the limitations in our study was the restrictions posed by sample size. We provided the $95 \%$ confidence interval values to provide quantitative assessment of the precision of the estimate. Although the current 1-month stroke and/or death rates seen following intracranial stent placement are encouraging, our study does not provide any information regarding the long-term results in regard to both clinical events and restenosis. Based on the results of the current study, the China Angioplasty and Stenting for Symptomatic Intracranial Severe Stenosis trial has been initiated and is an ongoing, prospective, multicenter randomized trial, which is being conducted in 8 sites intending to recruit 380 subjects (stent placement, 190; medical treatment alone, 190). ${ }^{28}$ The study aims to demonstrate a $10.7 \%$ absolute reduction in ipsilateral stroke and/or death during 12 months (assuming an event rate of $18 \%$ for medically treated patients ${ }^{3}$ and $7.3 \%$ for stenttreated patients ${ }^{19}$ ). The sample size provides $80 \%$ power with a 2 -sided test at the $5 \%$ level of significance and provides a $20 \%$ rate of lost follow-up.

\section{CONCLUSIONS}

The results of this prospective multicenter study demonstrated the outcome with safety in patients treated with Wingspan stent within 30 days, which suggested operators' experience at highvolume sites and strict patient selection are critical in reducing periprocedural complications and events.

\section{APPENDIX}

Principal investigators: L. Jiao, F. Ling, D. Wang

Participating clinical centers:

1) Xuanwu Hospital, Capital Medical University, Beijing: F. Ling, L. Jiao, P. Gao, F. Zhu, Y. Wang, Z. Wang, Y. Lu

2) Tangdu Hospital, Fourth Military Medical University, Xi'an: Z. Zhao, J. Yu

3) The 306 Hospital of the People's Liberation Army, Beijing: Y. Cai, Y. Cui

4) Henan Provincial People's Hospital, Zhengzhou: T. Li, L. Zhu, Z. Feng, Y. He

5) The 1st Affiliated Hospital of Harbin Medical University, Harbin: H. Shi, S. Xu, B. Zheng

6) Qilu Hospital of Shandong University, Ji'nan: W. Wu, S. Yin

7) The 2nd Affiliated Hospital of Guangzhou Medical College, Guangzhou: W. He, Y. He, S. Pu, B. Long

8) Tianjin Huanhu Hospital, Tianjin: L. Yin

9) Guangdong Province Hospital of Traditional Chinese Medicine, Guangzhou: S. Huang

10) Beijing Hospital of the Ministry of Health of China, Beijing: D. Wang, J. Liu.

\section{REFERENCES}

1. Wong KS, Li H. Long-term mortality and recurrent stroke risk among Chinese stroke patients with predominant intracranial atherosclerosis. Stroke 2003;34:2361-66 CrossRef Medline

2. Wang Y, Zhao X, Liu L, et al; CICAS Study Group. Prevalence and outcomes of symptomatic intracranial large artery stenoses and occlusions in China: the Chinese Intracranial Atherosclerosis (CICAS) study. Stroke 2014;45:663-69 CrossRef Medline

3. Chimowitz MI, Lynn MJ, Howlett-Smith H, et al; Warfarin-Aspirin Symptomatic Intracranial Disease Trial Investigators. Comparison of warfarin and aspirin for symptomatic intracranial arterial stenosis. N Engl J Med 2005;352:1305-16 CrossRef Medline

4. Fiorella D, Levy EI, Turk AS, et al. US multicenter experience with the Wingspan stent system for the treatment of intracranial atheromatous disease: periprocedural results. Stroke 2007;38:881-87 CrossRef Medline

5. Cruz-Flores S, Diamond AL. Angioplasty for intracranial artery stenosis. Cochrane Database Syst Rev 2006;CD004133 Medline

6. Ma N, Mo DP, Gao F, et al. Endovascular recanalization for chronic symptomatic middle cerebral artery total occlusion. J Neurointerv Surg 2013;5:e15 CrossRef Medline

7. Aghaebrahim A, Jovin T, Jadhav AP, et al. Endovascular recanalization of complete subacute to chronic atherosclerotic occlusions of intracranial arteries. J Neurointerv Surg 2014;6:645-48 CrossRef Medline

8. Chimowitz MI, Lynn MJ, Derdeyn CP, et al; SAMMPRIS Trial Investigators. Stenting versus aggressive medical therapy for intracranial arterial stenosis. N Engl J Med 2011;365:993-1003 CrossRef Medline

9. Derdeyn CP, Chimowitz MI, Lynn MJ, et al; Stenting and Aggressive Medical Management for Preventing Recurrent Stroke in Intracranial Stenosis Trial Investigators. Aggressive medical treatment with or without stenting in high-risk patients with intracranial artery stenosis (SAMMPRIS): the final results of a randomised trial. Lancet 2014;383:333-41 CrossRef Medline

10. SSYLVIA Study Investigators. Stenting of Symptomatic Atherosclerotic Lesions in the Vertebral or Intracranial Arteries (SSYLVIA): study results. Stroke 2004;35:1388-92 CrossRef Medline

11. Bose A, Hartmann M, Henkes H, et al. A novel, self-expanding, nitinol stent in medically refractory intracranial atherosclerotic stenoses: the Wingspan study. Stroke 2007;38:1531-37 CrossRef Medline

12. Jiang WJ, Xu XT, Jin M, et al. Apollo stent for symptomatic atherosclerotic intracranial stenosis: study results. AJNR Am J Neuroradiol 2007;28:830-34 Medline

13. Qureshi AI, Al-Senani FM, Husain S, et al. Intracranial angioplasty and stent placement after stenting and aggressive medical management for preventing recurrent stroke in intracranial stenosis (SAMMPRIS) trial: present state and future considerations. J Neuroimaging 2012;22:1-13 CrossRef Medline

14. Kernan WN, Ovbiagele B, Black HR, et al; American Heart Association Stroke Council, Council on Cardiovascular and Stroke Nursing, Council on Clinical Cardiology, and Council on Peripheral Vascular Disease. Guidelines for the prevention of stroke in patients with stroke and transient ischemic attack: a guideline for healthcare professionals from the American Heart Association/American Stroke Association. Stroke 2014;45:2160-236 CrossRef Medline

15. Fiorella D, Derdeyn CP, Lynn MJ, et al; SAMMPRIS Trial Investigators. Detailed analysis of periprocedural strokes in patients undergoing intracranial stenting in Stenting and Aggressive Medical Management for Preventing Recurrent Stroke in Intracranial Stenosis (SAMMPRIS). Stroke 2012;43:2682-88 CrossRef Medline

16. DasGupta A, Cai TT, Brown LD. Interval estimation for a binomial proportion. Statist Sci 2001;16:101-33 CrossRef

17. Miao Z, Song L, Liebeskind DS, et al. Outcomes of tailored angioplasty and/or stenting for symptomatic intracranial atherosclerosis: a prospective cohort study after SAMMPRIS. J Neurointerv Surg 2015;7:331-35 CrossRef Medline

18. Jiang WJ, Cheng-Ching E, Abou-Chebl A, et al. Multicenter analysis 
of stenting in symptomatic intracranial atherosclerosis. Neurosurgery 2012;70:25-30; discussion 31 CrossRef Medline

19. Jiang WJ, Yu W, Du B, et al. Outcome of patients with $\geq 70 \%$ symptomatic intracranial stenosis after Wingspan stenting. Stroke 2011; 42:1971-75 CrossRef Medline

20. Siddiq F, Chaudhry SA, Khatri R, et al. Rate of postprocedural stroke and death in SAMMPRIS trial-eligible patients treated with intracranial angioplasty and/or stent placement in practice. Neurosurgery 2012;71:68-73 CrossRef Medline

21. Nahab F, Lynn MJ, Kasner SE, et al; NIH Multicenter Wingspan Intracranial Stent Registry Study Group. Risk factors associated with major cerebrovascular complications after intracranial stenting. Neurology 2009;72:2014-19 CrossRef Medline

22. Chaturvedi S, Dumont AS. The learning curve for neuroendovascular procedures: how important is it? Neurology 2009;72:1974-75 CrossRef Medline

23. Derdeyn CP, Fiorella D, Lynn MJ, et al; Stenting and Aggressive Medical Management for Preventing Recurrent Stroke in Intracranial Stenosis Trial Investigators. Mechanisms of stroke after intracranial angioplasty and stenting in the SAMMPRIS trial. Neurosurgery 2013;72:777-95; discussion 795 CrossRef Medline
24. Zaidat OO, Klucznik R, Alexander MJ, et al; NIH Multi-center Wingspan Intracranial Stent Registry Study Group. The NIH registry on use of the Wingspan stent for symptomatic $70-99 \%$ intracranial arterial stenosis. Neurology 2008;70:1518-24 CrossRef Medline

25. Abou-Chebl A, Steinmetz H. Critique of "Stenting versus aggressive medical therapy for intracranial arterial stenosis" by Chimowitz et al in the New England Journal of Medicine. Stroke 2012;43:616-20 CrossRef Medline

26. Hussein HM, Emiru T, Georgiadis AL, et al. Assessment of platelet inhibition by point-of-care testing in neuroendovascular procedures. AJNR Am J Neuroradiol 2013;34:700-06 CrossRef Medline

27. Chaudhry SA, Watanabe M, Qureshi AI. The new standard for performance of intracranial angioplasty and stent placement after Stenting versus Aggressive Medical Therapy for Intracranial Arterial Stenosis (SAMMPRIS) trial. AJNR Am J Neuroradiol 2011;32: E214 CrossRef Medline

28. Gao P, Zhao Z, Wang D, et al. China Angioplasty and Stenting for Symptomatic Intracranial Severe Stenosis (CASSISS): a new, prospective, multicenter, randomized controlled trial in China. Interv Neuroradiol 2015;21:196-204 CrossRef Medline 